Elsevier

IJP 01914

\title{
A mathematical model to describe drug release from thin topical applications
}

\author{
William J. Addicks ${ }^{1}$, Gordon Flynn ${ }^{2}$, Norman Weiner ${ }^{2}$ and Rane Curl ${ }^{3}$ \\ ${ }^{\prime}$ E.I. DuPont de Nemours \& Co., Medical Products Department, Experimental Station, Wilmington, DE 19898 (U.S.A.), \\ ${ }^{2}$ College of Pharmacy, University of Michigan, Ann Arbor, MI 48109 (U.S.A.) \\ and ${ }^{3}$ Department of Chemical Engineering, University of Michigan, Ann Arbor, MI 48109 (U.S.A.)
}

(Received 28 December 1988)

(Modified version received 30 May 1989)

(Accepted 8 June 1989)

Key words: Topical; Vehicle; Thin application; Diffusion; Mathematical model

\section{Summary}

Release of a thin topical application of a drug is modeled by transient diffusion between the application, the underlying stratum corneum, and a receptor. Cases treated include sudden contact of the application with drug-free stratum corneum, and initially equilibrated application and stratum corneum. The method of solution is the numerical inversion of the analytic Laplace Transform of the exact solution, which has speed, accuracy and convenience advantages over classical analytical or numerical methods for coupled linear partial differential equations. The algorithm for long-time solutions in the time domain is also found, although this has no computational advantage over the direct numerical transform inversion.

\section{Introduction}

In studies involving the transdermal delivery of drugs across the skin, permeation experiments are usually performed under steady-state conditions, and the results are interpreted through the use of steady-state models. However, in the clinical setting, drug delivery occurs from applications which are only 20-50 $\mu \mathrm{m}$ in thickness (Flynn et al., 1985). Thus, even when a drug is formulated at high percentage composition, only a minute mass of drug is available for diffusion per unit area of the application. Under this condition, permeation is almost certainly a non-steady process. Two con-

Correspondence: W.J. Addicks, E.I. DuPont de Nemours \& Co., Medical Products Department, Experimental Station, E400/1255, Wilmington, DE 19898, U.S.A. certed, complex sets of events control the delivery of drugs across the skin in this situation. The initial kinetic factors of consequence are those that control movement of mass within the dosage form itself. Of at least equal importance are the factors that control mass transfer across the skin. A model describing drug delivery through the skin from a finite topical application should properly account for all these factors. Such a comprehensive model would aid in the design of in vitro experiments by clearly elucidating the relative importance of the various rate-influencing processes. In addition, the model would expedite the design of topical dosage forms, as it would enable formulators to predict the rate of drug delivery from a particular topical formulation.

Equations describing the diffusion of a drug through the skin from a thin topical application in 
which the drug is in solution were derived by Guy and Hadgraft (1980). That model is based on the assumption that the skin is initially devoid of drug. Although totally reasonable theoretically, this initial condition makes the model difficult to verify experimentally. This is so because it takes an appreciable time to spread the topical dosage form, for example, a gel, onto the membrane of study, place it in the diffusion chamber with the applied film of formulation intact and then set the experiment into motion. Moreover, air bubbles, which are usually present, must then be teased out of the cell before the experiment can proceed. Consequently significant time elapses between the application of the formulation to the membrane and the start of an experiment. During this time the drug diffuses into the membrane, leading to uncertainty as to how closely the boundary conditions of the model are experimentally met. This concern is particularly acute for cases in which the drug has a high membrane/application partition coefficient and/or is present in an ultra-thin application thickness and/or diffuses readily through the membrane, as all of these factors contribute to a large percentage of the contents of the application being delivered into the membrane in a short period. The time involved in preparing the membrane would not be expected to present problems in typical experiments involving intact skin where diffusional times through the statum corneum are long compared to preparation times. However, problems are likely to be encountered when one attempts to study permeation from thin applications through damaged skin or synthetic membranes.

This paper describes the derivation and solution of an equation that generalizes the Guy and Hadgraft model, which allows alternative initial conditions with respect to drug concentration in the application and the membrane to be considered. Equations for two initial conditions are specifically presented; one for the previously studied case in which the membrane is initially devoid of drug and another for the new case in which the drug is allowed to equilibrate between the application and the membrane prior to the start of an experiment. The latter is a useful initial condition with respect to in vitro experiments.
The solutions to the model are presented in the Laplace domain and only numerical inversion is required, rather than analytical or numerical solution of coupled partial differential equations.

\section{Derivation}

Assume that the diffusion of a drug from a topical dosage form takes place in accordance with the scheme depicted in Fig. 1. In this diagram, $C_{\mathrm{v}}$ and $C_{\mathrm{sc}}$ (not assumed to be uniform) are the drug's concentrations in the vehicle application and stratum corneum, $D_{v}$ and $D_{\mathrm{sc}}$ are the drug's diffusion coefficient in the vehicle application and the drug's effective diffusion coefficient in the stratum corneum, and $h_{\mathrm{v}}$ and $h_{\mathrm{sc}}$ are the thicknesses of the application and stratum corneum, respectively. The total thickness of the composite, $h$ is defined as $h_{\mathrm{v}}+h_{\mathrm{sc}} . K$ is the partition coefficient at the membrane/application interface, and is defined as $C_{\mathrm{sc}} / C_{\mathrm{v}}$. It is assumed that a sink condition $(C=0)$ exists at the membrane-receptor interface at all times. An equation is sought which describes the mass of drug being released into the sink as a function of time.

The equations that govern diffusion in the application phase and the stratum corneum phase are:

$D_{\mathrm{v}}\left(\frac{\partial^{2} C_{\mathrm{v}}}{\partial x^{2}}\right)=\frac{\partial C_{\mathrm{v}}}{\partial t}$

and

$D_{\mathrm{sc}}\left(\frac{\partial^{2} C_{\mathrm{sc}}}{\partial x^{2}}\right)=\frac{\partial C_{\mathrm{sc}}}{\partial t}$.

The generalized initial conditions for the problem are:

$$
\begin{array}{lll}
C_{\mathrm{v}}=C_{\mathrm{v} 0} \quad h>x>h_{\mathrm{sc}} & t=0 \\
C_{\mathrm{sc}}=C_{\mathrm{sc} 0} \quad h_{\mathrm{sc}}>x>0 & t=0
\end{array}
$$

The boundary conditions (for all time) are:

$$
\begin{array}{ll}
\frac{\partial C_{\mathrm{v}}}{\partial x}-0 & x-h \\
C=0 & x=0
\end{array}
$$




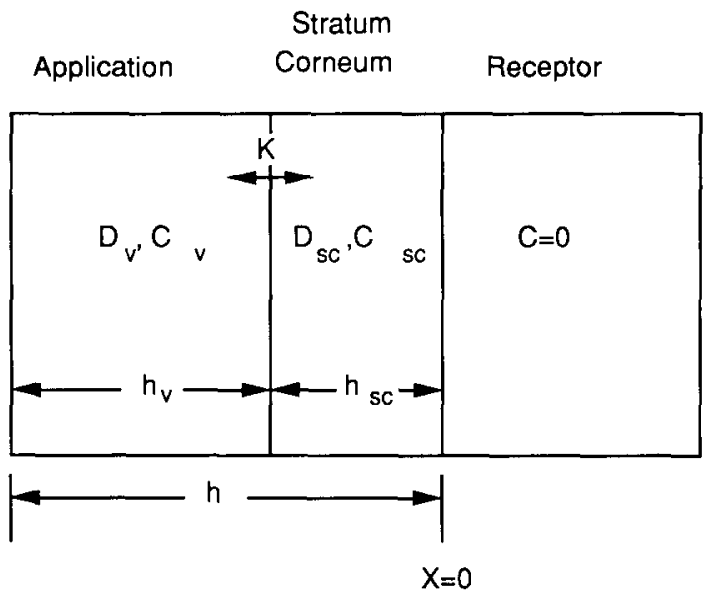

Fig. 1. Schematic representation of a topical application as it resides on the surface of the stratum corneum.

$K=\frac{C_{\mathrm{sc}}}{C_{\mathrm{v}}} \quad x=h_{\mathrm{sc}}$

$D_{\mathrm{v}}\left(\frac{\partial C_{\mathrm{v}}}{\partial x}\right)=D_{\mathrm{sc}}\left(\frac{\partial C_{\mathrm{sc}}}{\partial x}\right) \quad x=h_{\mathrm{sc}}$

These equations are conveniently simplified by the method of Laplace Transforms. Guy and Hadgraft (1980) show the manipulations for the case $C_{\mathrm{sc} 0}=0$. The same procedures, with $C_{\mathrm{sc} 0} \neq 0$, are shown by Addicks (1988), and yield:

$$
\begin{aligned}
\frac{M^{*}}{M_{\infty}}= & {\left[\sqrt{D_{\mathrm{sc}}} /\left\{\left(C_{\mathrm{v} 0} h_{\mathrm{v}}+C_{\mathrm{sc} 0} h_{\mathrm{sc}}\right) s^{3 / 2}\right\}\right] } \\
& \times\left[\left\{\left(C_{\mathrm{v} 0} K-C_{\mathrm{sc} 0}\right) \sinh \sqrt{\frac{s}{D_{\mathrm{v}}}} h_{\mathrm{v}}\right\}\right] \\
& \left\{K \left(\frac{D_{\mathrm{sc}}}{D_{\mathrm{v}}} \cdot \cosh \sqrt{\frac{s}{D_{\mathrm{v}}}} h_{\mathrm{v}} \cdot \cosh \sqrt{\frac{s}{D_{\mathrm{sc}}}} h_{\mathrm{sc}}\right.\right. \\
& \left.\left.+\sinh \sqrt{\frac{s}{D_{\mathrm{v}}}} h_{\mathrm{v}} \cdot \sinh \sqrt{\frac{s}{D_{\mathrm{sc}}}} h_{\mathrm{sc}}\right)^{1 / 2}\right\} \ldots+\ldots \\
& \ldots+\ldots\left[\sqrt{D_{\mathrm{sc}}} /\left\{\left(C_{\mathrm{v} 0} h_{\mathrm{v}}+C_{\mathrm{sc} 0} h_{\mathrm{sc}}\right) s^{3 / 2}\right\}\right] \\
& \times\left[\left\{C _ { \mathrm { sc } 0 } \left(\sinh \sqrt{\frac{s}{D_{\mathrm{v}}}} h_{\mathrm{v}} \cdot \cosh \sqrt{\frac{s}{D_{\mathrm{sc}}}} h_{\mathrm{sc}}\right.\right.\right.
\end{aligned}
$$

$$
\begin{aligned}
& \left.\left.+K \sqrt{\frac{D_{\mathrm{sc}}}{D_{\mathrm{v}}}} \cdot \sinh \sqrt{\frac{s}{D_{\mathrm{sc}}}} h_{\mathrm{sc}} \cdot \cosh \sqrt{\frac{s}{D_{\mathrm{v}}}} h_{\mathrm{v}}\right)\right\} / \\
& \left\{K \sqrt{\frac{D_{\mathrm{sc}}}{D_{\mathrm{v}}}} \cdot \cosh \sqrt{\frac{s}{D_{\mathrm{v}}}} h_{\mathrm{v}} \cdot \cosh \sqrt{\frac{s}{D_{\mathrm{sc}}}} h_{\mathrm{sc}}\right. \\
& \left.\left.+\sinh \sqrt{\frac{s}{D_{\mathrm{v}}}} h_{\mathrm{v}} \cdot \sinh \sqrt{\frac{s}{D_{\mathrm{sc}}} h_{\mathrm{sc}}}\right\}\right]
\end{aligned}
$$

where $M^{*}$ and $M_{\infty}$ are the mass transferred up to time $t$ and the initial drug loading, respectively, and $s$ is the Laplace Transform variable.

For reasons previously mentioned it is useful to have an expression for the situation in which the drug is initially in equilibrium with both the membranc and application. In this case, the model assumes that prior to the start of an experiment the application is placed onto the membrane until a state of equilibrium is reached. This is not a realistic situation for drug delivery clinically but it does describe the simplest case for preparing a membrane for mass transport study using in vitro diffusion cell methodology. For this case the following initial condition applies:

$C_{\mathrm{sc} 0}=K \cdot C_{\mathrm{v} 0} \quad h>x>0$

This initial condition in Eqn. 3 gives:

$$
\begin{aligned}
\frac{M^{*}}{M_{\infty}}= & \left.\left\{\sqrt{D_{\mathrm{sc}}} K\right\} /\left\{\left(h_{\mathrm{v}}+h_{\mathrm{sc}} K\right)\left(s^{3 / 2}\right)\right\}\right] \\
& \times\left[\left\{\sinh \sqrt{\frac{s}{D_{\mathrm{v}}}} h_{\mathrm{v}} \cdot \cosh \sqrt{\frac{s}{D_{\mathrm{sc}}}} h_{\mathrm{sc}}\right.\right. \\
& +\left(K \sqrt{\frac{D_{\mathrm{sc}}}{D_{\mathrm{v}}}} \cdot \sinh \sqrt{\frac{s}{D_{\mathrm{sc}}}} h_{\mathrm{sc}}\right. \\
& \left.\left.\cdot \cosh \sqrt{\frac{s}{D_{\mathrm{v}}}} h_{\mathrm{v}}\right)\right\} / \\
& \left\{\left(K \sqrt{\frac{D_{\mathrm{sc}}}{D_{\mathrm{v}}}} \cdot \cosh \sqrt{\frac{s}{D_{\mathrm{v}}}} h_{\mathrm{v}}\right.\right.
\end{aligned}
$$




$$
\begin{aligned}
& \left.\cdot \cosh \sqrt{\frac{s}{D_{\mathrm{sc}}}} h_{\mathrm{sc}}\right)+\sinh \sqrt{\frac{s}{D_{\mathrm{v}}}} h_{\mathrm{v}} \\
& \left.\left.\cdot \sinh \sqrt{\frac{s}{D_{\mathrm{sc}}}} h_{\mathrm{sc}}\right)\right]
\end{aligned}
$$

For the case in which the membrane is initially free of drug, the following initial condition applies:

$$
C_{\mathrm{sc} 0}=0 \quad h_{\mathrm{sc}}>x>0, \quad t=0
$$

This initial condition in Eqn. 3 gives:

$$
\begin{aligned}
\frac{M^{*}}{M_{\infty}}= & {\left[\left\{\sqrt{D_{\mathrm{sc}}} K\right\} /\left\{\left(h_{\mathrm{v}}\right)\left(s^{3 / 2}\right)\right\}\right] } \\
& \times\left[\left\{\sinh \sqrt{\frac{s}{D_{\mathrm{v}}}} h_{\mathrm{v}}\right\}\right] \\
& \left\{\left(K \sqrt{\frac{D_{\mathrm{sc}}}{D_{\mathrm{v}}}} \cdot \cosh \sqrt{\frac{s}{D_{\mathrm{v}}}} h_{\mathrm{v}}\right.\right. \\
& \left.\cdot \cosh \sqrt{\frac{s}{D_{\mathrm{sc}}}} h_{\mathrm{sc}}\right)+\sinh \sqrt{\frac{s}{D_{\mathrm{v}}}} h_{\mathrm{v}} \\
& \left.\left.\cdot \sinh \sqrt{\frac{s}{D_{\mathrm{sc}}}} h_{\mathrm{sc}}\right\}\right]
\end{aligned}
$$

which is identical (except for the different nomenclature) to that found by Guy and Hadgraft (1980).

\section{Discussion}

In order to be used, Eqns. 4 and 5 must be inverted from functions of $s$ to functions of $t$. Tables containing functions and their Laplace transformations have been compiled and are typically used for this purpose. However, Eqns. 4 and 5 are not found in the published literature, and thus inversion must be performed via numerical methods. Because of this, Guy and Hadgraft (1980) attempted only to find long-term and short-term

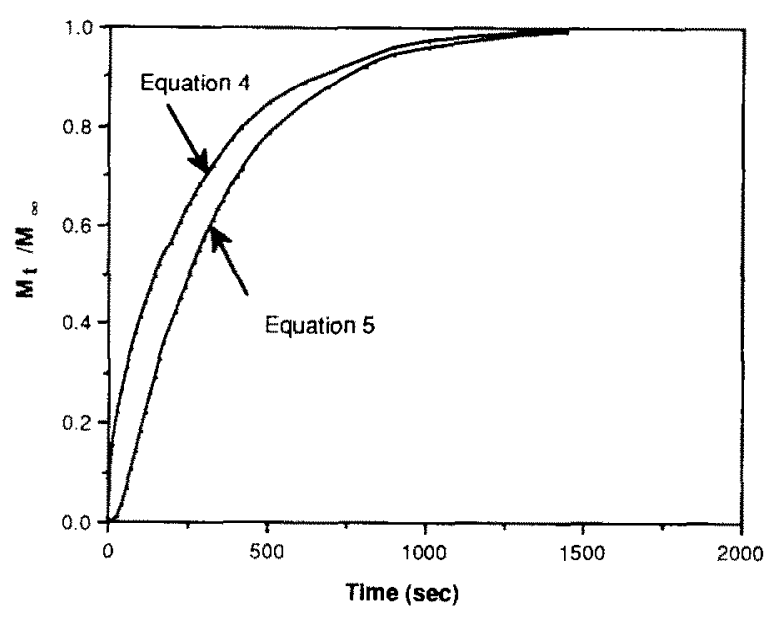

Fig. 2. Relative mass versus time profiles as predicted by Eqns. 4 and 5. Parameter values used in the simulations are found in the text.

solutions. Numerical inversion is today, however, easily accomplished with the use of the fast Fourier transform, which is available in numerous computer libraries. The validity of this method was demonstrated in a recent paper where the analytical solution and an orthogonal solution to an equation describing a fixed-bed adsorber were compared to the solution obtained through the use of the fast Fourier transforms (Chen and Hsu, 1987). The 3 solutions were identical, and the fast Fourier transform method was found to be two orders of magnitude faster than the orthogonal collocation method. This method is a new and extremely powerful tool for the solution of any diffusional problem encountered in biological and pharmaceutical systems.

Fig. 2 shows the solutions of Eqns. 4 and 5 for the case in which the drug's membrane diffusivity is $2.0 \times 10^{-6} \mathrm{~cm}^{2} / \mathrm{s}$, the diffusivity in the vehicle is $7.0 \times 10^{6} \mathrm{~cm}^{2} / \mathrm{s}$, the drug's membrane/vehicle partition coefficient is 0.5 , and the thicknesses of the membrane and vehicle are $250 \mu \mathrm{m}$ and $75 \mu \mathrm{m}$, respectively. These parameter values are similar to those that might be encountered in the case of drug diffusion from a topical dosage form through a silicone rubber membrane, a membrane used as a model membrane in some of our work. As expected, the situation in which the drug is initially in equilibrium between membrane and 
vehicle (Eqn. 4) is associated with a much faster initial rate of drug delivery than when the membrane is initially free of drug (Eqn. 5). This early unloading of drug has been termed a "burst effect" in some literature. A lag time associated with the establishment of the concentration gradient across the membrane characterizes the situation in which the membrane is initially devoid of drug.

Guy and Hadgraft (1980) found the long-time solution in the time domain, for Eqn. 5, by finding the small $s$ algebraic limit. This procedure is not entirely correct. The long-time solution is obtained by finding the dominant (small) pole of the transfer function, as described by, e.g. Churchill (1944). To illustrate for just Eqn. 5: the poles of Eqn. 5 are only real and non-positive, and lie as $s=0$ and the roots of:

$\tan \sqrt{\frac{-s}{D_{\mathrm{v}}}} h_{\mathrm{v}} \times \tan \sqrt{\frac{-s}{D_{\mathrm{sc}}}} h_{\mathrm{sc}}=K \sqrt{\frac{D_{\mathrm{sc}}}{D_{\mathrm{v}}}}$

Two comparisons of the long-time and exact solutions are shown in Figs. 3 and 4. The parameters correspond to the cases considered by Guy and Hadgraft (1980), and are:

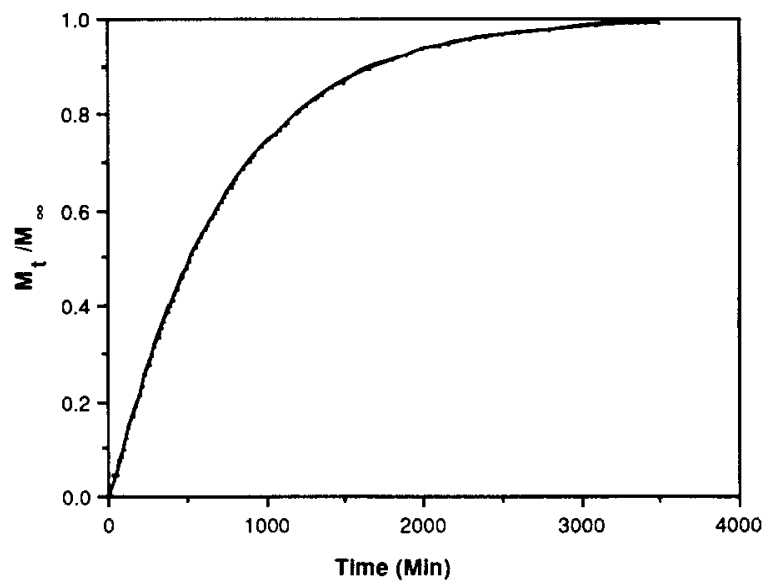

Fig. 3. Relative mass versus time profiles as predicted by the exact (Eqn. 5) and long-time approximate solutions for diffusion through normal skin (case 1 as described in text). The simulations are virtually coincident.

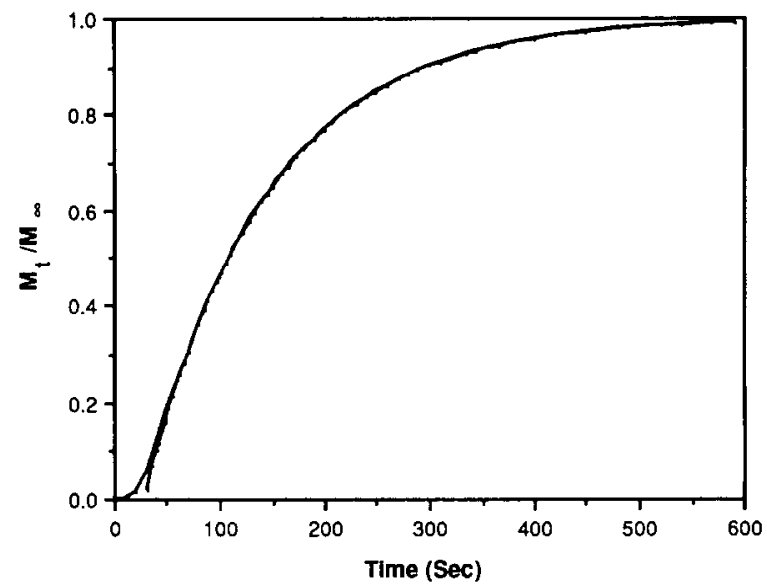

Fig. 4. Relative mass versus time profiles as predicted by the exact (Eqn. 5) and long-time approximate solutions for diffusion through abraded skin (case 2 as described in text). The simulations are virtually coincident.

\begin{tabular}{lll} 
Case & $\mathbf{1}$ & $\mathbf{2}$ \\
\hline Figure & 3 & 4 \\
$D_{\text {sc }}\left(\mathrm{cm}^{2} / \mathrm{s}\right)$ & $1 \times 10^{-10}$ & $1 \times 10^{-6}$ \\
$D_{\mathrm{v}}$ & $1 \times 10^{-6}$ & $1 \times 10^{-6}$ \\
$K$ & 0.5 & 1.0 \\
$h_{\mathrm{sc}}(\mu \mathrm{m})$ & 10 & 150 \\
$h_{\mathrm{v}}$ & 20 & 20 \\
$-s\left(\mathrm{~h}^{-1}\right)$ & 0.0830 & 30.7 \\
\hline
\end{tabular}

Case 1 corresponds to drug diffusion from a thin topical application into normal skin, and Case 2 to diffusion into abraded skin. The long-time solutions are essentially identical to the exact solution except (of course) at short times. The long-time expressions are easily found by the partial fraction expansion of Eqn. 5. Retaining only the first exponential term yields, for Case 1:

$\frac{M^{*}}{M_{\infty}}=1-1.038 \exp (-0.0830 t)$

and for Case 2:

$\frac{M^{*}}{M_{\infty}}=1-1.266 \exp (-30.7 t)$

which are plotted in Figs. 3 and 4 for the region of time for which they are positive. 
A more complete partial fraction expansion is readily done by finding more (larger) roots of Eqn. 5, and using the methods of partial fraction expansions to determine the coefficients. This is readily implemented with a computer, but has hardly any advantage over the numerical inversion of the complete Laplace Transform unless an algebraic expression in the time domain is desired.

In summary, a general solution in terms of Laplace transforms has been obtained for the problem of drug diffusion from a thin topical application through a resistant membrane. Solutions have been presented for the case in which the membrane is initially free of drug as well as for the case in which the drug is initially in equilibrium with respect to the vehicle and membrane, a situation which allows one to more easily study transport from thin topical applications through highly permeable membranes. These exact solutions have been numerically inverted, and the former case has been compared to long-time approximate solutions. However, the approximate solution method has no advantage over the direct numerical inversion of the Laplace Transform of the solution, in either time or convenience.

\section{Acknowledgements}

The authors gratefully acknowledge S. Ramanathan for providing the computer program that made it possible to invert the Laplace transforms. W.J.A. would like to thank the United States Pharmacopeial Convention, Inc. for providing him with a fellowship.

\section{References}

Addicks, W.J., Drug Release from Thin Topical Applications, Ph.D. thesis, University of Michigan, Ann Arbor, 1988, p. 104.

Chen, T.L. and Hsu, J.T., Prediction of breakthrough curves by the application of fast Fourier transforms. AlChE J., 33 (1987) 1387.

Churchill, R.V., Modern Operational Mathematics in Engineering, McGraw Hill, New York, NY, 1944.

Flynn, G., Topp, E. and Amidon, G., Physicochemical aspects of drug delivery to and via the skin. In Topics in Pharmaceutical Science, Elsevier, Amsterdam, 1985, pp. 313-328.

Guy, R. and Hadgraft, J., A theoretical description relating skin permeation to the thickness of the applied medicament. Int. J. Pharm., 6 (1980) 321-332. 